\title{
Maternal Uniparental Disomy for Chromosome 14
}

\author{
D.A. Coviello', E. Panucci ${ }^{1}$, M.M. Mantero ${ }^{2}$, C. Perfumo ${ }^{2}$, M. Guelfi $^{3}$, \\ C. Borrone ${ }^{4}$, F. Dagna Bricarelli ${ }^{2}$ \\ 'Istituto di Biologia e Genetica (IB/G), Università di Genova, Italy; ${ }^{2}$ Centro di Genetica \\ Umana, E.O. Ospedali Galliera, Genova, Italy; ${ }^{3}$ stituto di Clinica Ortopedica, Università \\ di Genova, Italy; ${ }^{4}$ Divisione di Pediatria II, Istituto G. Gaslini, Genova, Italia
}

\begin{abstract}
A girl carrying a de novo balanced 13-14 robertsonian translocation showed a clinical phenotype with severe hypotonia, hyperextensible joints, frontal bossing, asymmetric face, no mental retardation, severe scoliosis and motor delay. In situ hybridization analysis on chromosome spreads revealed the presence of the two centromeres in the rearranged chromosomes. Molecular analysis on genomic DNA showed the presence in the proposita of two chromosomes 14 of maternal origin and no chromosome 14 from the father indicating a maternal monocentric uniparental disomy for chromosome 14 (mUPD14). Our patient shows several similarities with other reported cases of mUPD14, suggesting imprinting of a region(s) of chromosome 14 and defining a possible mUPD 14 Syndrome.
\end{abstract}

Key words: Uniparental disomy; Chromosome 14; Robertsonian translocation, Imprinting

\section{INTRODUCTION}

Uniparental disomy (UPD) defines the presence of two homologous chromosome derived from one parent in a diploid offspring, whereas the inheritance of two copies of the identical homologous chromosome from one parent is called uniparental isodisomy [1]. The abnormal phenotype may be due to the loss of contribution of genetic information from one parent due to imprinted regions or to the differential expression of the parental alleles in uniparental disomy and may be due to reduction to homozygosity o the genetic information in uniparental isodisomy [2]. We report on the occurrence of maternal UPD for chromosome 14 (mUPD14) in a girl with a de novo robertsonian translocation $t(13 ; 14)$. Four previous cases of mUPD14 have been reported $[3,4,5,6]$. 
Comparison of the clinical features of the four cases with the one currently described uncovers some common findings which might result from maternally imprinted genes that cause the mUPD14 syndrome.

\section{Case history}

The proposita is a girl carrying a de novo balanced 13-14 translocation detected during cytogenetic prenatal diagnosis requested for psychological reasons by a 32 old woman. The proposita, born after 40 week gestation, had an angar vame of $z$ at 9 min and a cyanotic episode within few minutes; she was initially evaluated in the newborn period because of severe hypotonia, hyperextensible joints, minor facial dysmorphisms: frontal bossing and asymmetric face; at the age of 15 months, the patient showed no mental retardation, a severe scoliosis and motor delay.

\section{MATERIALS AND METHODS}

\section{Cytogenetic analysis}

The amniocentesis was performed at the 17 th week of gestation, the amniocytes were cultured using the in situ method. Chromosome spreads were analysed using FQ and CBG banding. Peripheral blood cultures, from the newborn and her parents, were set up according to a standard protocol. The probes used for in situ hybridization were D13Z1/D21Z1 (chromosomes 13 and 21 centromeres) and D14Z1/D22Z1 (chromosomes 14 and 22 centromeres). All probes were obtained from Oncor, Inc. (Gaithersburg, MD, USA). Fluorescence in situ hybridization using biotinilated probes was performed with the method of Pinkel et al. as described [7].

\section{Nucleic acid studies}

Genomic DNA was extracted from peripheral blood samples as described elsewhere [8]. PCR reactions were performed using Taq Polymerase (promega), and the reactions were performed according to the manufacturer's conditions in a total volume of $25 \mu \mathrm{l}$. Either inclusion of $\left[\alpha^{33}\right] \mathrm{P}-\mathrm{dATP}$ in the PCR reaction or use of $5^{\prime}-$ end labelled $\left(\left[\gamma^{32} \mathrm{P}\right]-\mathrm{dATP}\right)$ primers were used to obtain radioactive fragments. Amplification products were run on a 7\% acrylamide sequencing gel and dried gels were exposed overnight with Kodak XOMAT AR films. Molecular analysis to detect parental origin of chromosomes 13 and 14 was performed using microsatellite markers (chromosome 13: D13S221, D13S174, D13S153; chromosome 14: D14S64, D14S72 and MYH7 locus for MYOI and MYOII markers). Non paternity was tested using microsatellic markers of chromosomes 1,11 and 15: D1S249, D11S935, and D15S108.

\section{RESULTS}

Karyotype of the parents was normal. Cytogenetic study performed on amniocytes revealed a robertsonian translocation $113 ; 14$ and in situ hybridization analysis revealed the presence of two centromeres in the rearranged chromosome indicating that no loss 
of long arms occurred. At birth the peripheral blood of the newborn confirmed the robertsonian translocation $13 ; 14$, but the clinical phenotype indicated genetic imbalance and UPD was cheked for chromosomes 13 and 14. The results of molecular studies showed the presence of two chromosomes 14 of maternal origin and no chromosome 14 from the father indicating a maternal monocentric uniparental disomy for chromosome 14 (mUPD14). Non paternity was excluded by using markers from chromosomes 1,11 and 15 .

\section{DISCUSSION}

This is the fifth case of mUPD14 reported in patients with balanced 13-14 translocation; three previous reported cases were maternal, two "de novo" [5,7], one inherited [3]; one additional case was maternal uniparental isodisomy 14 [4].

One possible mechanism that could explain mUPD14 is the fertilisation of a maternal gamete containing a normal 14 and a chromosome with a translocation $\mathrm{t}(13 \mathrm{q}-14 \mathrm{q})$ with a paternal gamete nullisomic for chromosome 14 . A second and most likely hypothesis is that the maternal contribution to the zygote was the $\mathrm{t}(13 ; 14)$ chromosome and a normal chromosome 14 while the father normally contributed a chromosome 13 and 14; shortly after fertilization, the zygote, which was initially trisomic for chromosome 14 , lost the paternal chromosome 14 to become mUPD14.

Clinical phenotypes of all maternal UPD14 reported cases, including our patient, show several similarities: hypotonia, hyperextensible joints, minor facial dysmorphisms, severe scoliosis and motor delay. Only one case of paternal UPD14 was reported [9] and the phenotype appeared different.

Our patient is predominately heterodisomic suggesting that these common findings could be due to imprinting of a region(s) of chromosome 14 rather than homozygosity of recessive alleles, defining a possible mUPD14 Syndrome.

The reports of UPD rise new problems in cytogenetic evaluation of balanced translocations in genetic counselling of prenatal diagnosis; extensive studies of this phenomenon both in patients and in the phenotypically normal population carrying a balanced translocation will be necessary to establish a relationship between genotype and clinical features.

\section{REFERENCES}

1. Engel E: A new genetic concept: uniparental disomy and its potential effect, isodisomy. Am $\mathbf{J}$ Med Genet 1980; 6: 137-143.

2. Hall JG: Genomic imprinting: review and relevance to human disease. Am J Hum Genet 1990; 46: 857-873.

3. Temple IK, Cockwell A; Hassold T, Pettay D, Iacobs P: Maternal uniparental disomy for chromosome 14. J Med Genet 1991, 28: 511-514.

4. Pentao L; Lewis RA, Ledbetter DII, Patel PI, Lupski JR: Maternal uniparental disomy for chromosome 14: association with autosomal recessive rod monochromacy. Am J Hum Genet 1992; 50: 690-699. 
5. Antonarakis SE, Blouin JL, Maher J, Avramopopulus D, Thomas G, Talbot CC Jr: Maternal uniparental disomy for human chromosome 14, due to loss of a chromosome 14 from somatic cells with $\mathrm{t}(13 ; 14)$ trisomy 14 . Am J Hum Genet 1993; 52: 1145-1152.

6. Healey SD, Powell F, Battersby M, Chenevix-Trench G, McGill J: Distinct phenotype in maternal uniparental disomy of chromosome 14. Am J Med Genet 1994; 51: 147-149.

7. Pinkel D, Straume T, Gray JW: Cytogenetic analysis using quantitative, high-sensitivity, fluorescence hybridization. Proc Natl Acad SCI 1988; 83: 2934-2938.

8. Coviello DA, Bertolini S, Masturzo P, Chisellini M, Tiozzo R, Zambelli F, Stefanutti C, Torcia $\mathrm{F}$, Pachì $\mathrm{A}$, Calandara S: Chorionic DNA analysis for the prenatal diagnosis of familial hypercholesterolemia. Hum Genet 1993; 92: 424-426.

9 Wang J-CC, Passage MB, Yen PH, Shapiro LJ, Mohandas TK: Uniparental heterodisomy for chromosome 14 in a phenotypically abnormal familial balanced 13/14 robertsonian translocation carrier. Am J. Hum Genet 1991; 48:1069-1074.

Correspondence: D.A. Coviello, Istituto di Biologia e Genetica (IBIG), Università di Genova, Italy. 